\title{
Development of molecular markers for genetic male sterility in Gossypium hirsutum
}

\author{
Xuehui Feng • Don Keim • Humphrey Wanjugi • \\ Issa Coulibaly $\cdot$ Yan Fu · John Schwarz • \\ Scott Huesgen $\cdot$ Seungho Cho
}

Received: 13 January 2015/ Accepted: 26 May 2015/Published online: 7 June 2015

(C) The Author(s) 2015. This article is published with open access at Springerlink.com

\begin{abstract}
Genetic male sterility (GMS) in cotton mediated by two homozygous recessive genes, $m s 5 m$ $s 5$ and $m s 6 m s 6$, is expressed as non-dehiscent anthers and unviable pollen grains. Sequence analysis on $m s 5$ and $m s 6$ loci in Gossypium hirsutum was conducted to reveal genomic variation at these two loci between GMS and wild-type G. hirsutum inbred lines, and sequence polymorphism linked to ms 5 on A12 and $m s 6$ on D12 was revealed. A haplotype marker set that consisted of four SNPs targeting both $m s 5$ and $m s 6$ gene regions was developed and validated for association with GMS in cotton. Predictability of GMS phenotype by this haplotype SNP set was over $99 \%$. GMS haplotype marker set can serve as a highthroughput molecular breeding tool to select GMS individuals and improve hybrid production efficiency.
\end{abstract}

Keywords Genetic male sterility $(\mathrm{GMS}) \cdot$ Hybrid cotton · Molecular markers · Haplotype $\cdot$ Phenotype prediction

Xuehui Feng, Don Keim and Humphrey Wanjugi have contributed equally to this work.

X. Feng · D. Keim · H. Wanjugi · I. Coulibaly

Y. Fu · J. Schwarz $\cdot$ S. Huesgen $\cdot$ S. Cho $(\square)$

Monsanto Company, 800 N. Lindbergh Blvd,

St. Louis, MO 63141, USA

e-mail: seungho.cho@monsanto.com

\section{Introduction}

Genetic male sterility (GMS) in cotton occurs in a form of non-dehiscent anthers and unviable pollens when reproductive development process fails due to possible loss of nuclear gene functions required for pollen development. Unlike cytoplasmic male sterility, genes related to GMS phenotypes can be easily transferred to various genetic backgrounds by routine crossing practice and allow the trait to be inherited at full penetrance in successive generations. There have been 19 different GMS genes, Msl to Ms19, identified in tetraploid cotton species (summarized by Chen et al. 2009), and are classified by genetic characterization and multigenic nature such as single dominant gene (Ms4), single recessive gene $(m s 2)$ and paired recessive genes ( $m s 5$ and $m s \sigma$ ). Single recessive genes $m s l$ and $m s 3$ reportedly conferred partial genetic male sterility (Justus and Leinweber 1960; Justus et al. 1963). In case of $m s 3$, it produced fertile flowers more often in greenhouse compared with field condition (Justus et al. 1963). In contrast, GMS conferred by a single recessive gene, $m s 2$, and paired recessive genes, $m s 5$ and $m s 6$, conferred stable male sterility (Richmond and Kohel 1961). Because GMS maintainers need to be fertile, recessive genetic mechanisms with complete male sterility especially mediated by $m s 5$ and $m s 6$ have been widely used in hybrid cotton production (Basu 1996).

Cytoplasm male sterility (CMS) technology is also available to produce hybrid cotton. The first 
cytoplasmic male sterility was developed by transferring diploid A2 genome of Gossypium arboreum to cytoplasm of Gossypium anomalum carrying diploid B1 genome. Male sterile plants were produced when CMS inducer was pollinated by pollens of $G$. arboreum, and fertility was restored with G. anomalum pollen (Meyer and Meyer 1965; Meyer 1969). Because of incomplete or unstable male sterility and undesirable traits in CMS lines generated by $G$. arboreum and $G$. anomalum cytoplasm system, alternative CMS system called CMSD-2 was created by transferring genomes of commercial cotton (G. hirsutum) to Gossypium harknessii cytoplasm (Meyer 1973, 1975). Restorer of CMSD-2 system was created by transferring a restorer gene, Rfl, in G. harknessii to $G$. hirsutum genome (Meyer 1975). Cytoplasm of Gossypium trilobum carrying diploid D8 genome was also used to develop CMS system known as CMS-D8 (Stewart 1992), and Rf2 in D8 genome of $G$. trilobum was found to restore fertility (Zhang and Stewart 2001). Rfl functioning sporophytically was found to be nonallelic to $R f 2$ which functions gametophytically, and these two genes were linked on chromosome LGD08 which is same to D05 (Zhang and Stewart 2001, 2004; Liu et al. 2003; Feng et al. 2005; Wang et al. 2007; Yin et al. 2006). In spite of high male sterility frequency close to $100 \%$ by CMS, broader application of CMS to hybrid production has been limited due to narrow genetic choices of CMS and restorer line combination and cytoplasmic effect on potential yield drag and CMS stability (Meyer 1969; Meyer and Meyer 1965; Bhale and Bhat 1990; Schnable and Wise 1998).

The sources of $m s 5$ and $m s 6$ genes were speculated to be G. tomentosum or progeny from an interspecific cross with $G$. hirsutum. A nectariless $\mathrm{F}_{3}$ individual from a cross of Stoneville $20 \times$ nectariless $G$. tomentosum was crossed with a paternal parent, Empire WR. From this cross, a nectariless $F_{3}$ individual was crossed with Gregg. An $\mathrm{F}_{2}$ individual with nectariless phenotype was crossed to Lankart 57 and a single male sterile individual with nectariless phenotype was identified in their $F_{2}$ progenies (Weaver 1968). Recessive genetic inheritance of GMS trait was confirmed by backcrossing fertile progenies from the sterile individual against paternal ancestors. Assuming that there has been no spontaneous mutation occurring during crossing and population advancement, emergence of male sterility within $F_{3}$ progenies from interspecific cross between $G$. hirsutum and $G$. tomentosum might be due to interaction between two genes originated from two different tetraploid species. The recessive allele of either $m s 5$ or $m s 6$ originated from $G$. tomentosum might have interacted with the recessive allele that already existed in G. hirsutum to express male sterility. Although unlikely, the chance of spontaneous mutation at any stage throughout the interspecific and intraspecific crosses cannot be ruled out and can be considered as a possible source of GMS until disproven.

Genetic map positions of GMS genes were revealed by linkage mapping using SSR markers (Chen et al. 2009). Two Chinese GMS source lines, Lang-A carrying a recessive gene, $m s 15$, and Zhongkang-A carrying two recessive genes, $m s 5$ and $m s 6$, were crossed to Hai7124 carrying homozygous dominant alleles of both $m s 5$ and $m s 6$ first. Their $\mathrm{F}_{1}$ progenies were backcrossed to sterile parents to generate mapping populations in which only one of the two genes was segregating. Authors mapped each gene independently to chromosomes 12 ( $m s 5$ and $m s 15$ ) and 26 (ms 6 ) and claimed that $m s 5$ and $m s 15$ are two different genes on chromosome 12 with a distance of $6 \mathrm{cM}$ between loci. Rhyne (1991) reportedly mapped $m s 8$ and $m s 9$ on chromosome 12 and 26, respectively, as well. It is uncertain whether they are related to $m s 5$ and $m s 6$ as a test cross between these two genetic sources has not been attempted.

Although molecular and biochemical mechanisms of male sterility caused by ms $5 m s 5$ and ms $6 m s 6$ are not fully understood, analogous studies conducted in other species could be used to predict potential mechanism(s) that cause pollen development failure in cotton. In Arabidopsis, causes of failure in male gamete development were classified into four cases: (1) non-functioning stamen resulting in deformed anthers, (2) failure in microsporogenesis, (3) lack of pollen-coating agent, tryphine, and (4) non-dehiscent anthers (Okada and Shimura 1994). Observation of stamen and pollens from male sterile flowers indicated that all four cases appear to contribute to the sterility phenotype in cotton. Additional molecular mechanisms related to pollen development and mutant phenotypes due to genetic mutations on metabolic pathways are well characterized in model species (reviewed by Wilson and Zhang 2009; Okada and Shimura 1994; Chaudhury 1993). Genes of other functions but which are still associated with pollen 
development in Arabidopsis have been well characterized at the molecular level which include the following: $M S 1$, a PHD-finger family of transcription factor related to microsporogenesis (Wilson et al. 2001; Ito et al. 2007), MYB transcription factor related to dehiscence of pollen and pollen wall development (Steiner-Lange et al. 2003; Preston et al. 2004), MS2, elongation/condensation complex presumably related to pollen wall formation (Aarts et al. 1997), AtPTEN, tumor suppressor homolog related to pollen cell death after mitosis (Gupta et al. 2002). With in-depth wholegenome sequence data available, reverse genetic approaches can be used to further identify candidate genes associated with genetic male sterility in cotton. To characterize the genetic and genomic characteristics of the GMS in cotton, we conducted genomic analysis at $m s 5$ and $m s 6$ loci. Our study revealed genomic variation at both loci and also within candidate genes possibly associated with genetic male sterility in cotton. By SNP markers designed to detect genomic variation at both loci, a haplotype for GMS and predictability of GMS phenotype by SNP haplotyping were determined.

\section{Materials and methods}

\section{Genetic characterization of GMS}

Segregating populations for genetic study of GMS were generated from a cross between DPGh98018 and DPGh04651. DPGh98018 was used as a full fertile male parent, and DPGh04651 was used as a female parent presumably carrying multiple recessive GMS genes. Multiple recessive genetic inheritance patterns of GMS in DPGh04651 were observed within progenies generated by multiple generations of selfing in nursery in Scott, Mississippi during 2004-2005 season (data not provided). Male sterility of each plant was determined by monitoring presence of non-dehiscent anther in five flowers at anthesis over a month. In addition, flower abortion was also observed to confirm male sterility. Plants were scored as fertile if at least one dehiscent anther was visible. The same method was applied to phenotype all genetic materials used in our study. Based on inheritance patterns of GMS within progenies of DPGh04651, two recessive genes presumably $m s 5$ and $m s 6$ were speculated to confer GMS in DPGh04651. All genetic materials used in our study were prepared based on GMS mechanism by paired $m s 5$ and $m s 6$ genes. Sibcrossing between fertile and sterile $F_{2}$ progenies from a cross between DPGh98018 and DPGh04651 was made to produce sibcross $F_{1}$ progenies in the greenhouse in 2004 . Sibcross $F_{1}$ progenies were planted in greenhouse, and male sterility was phenotyped by each sibcrossing event. Fertile $F_{1}$ progenies were advanced to sibcross $\mathrm{F}_{2}$ populations by selfing. Segregation pattern of male sterility was monitored in each sibcross $F_{2}$ population independently, and sibcross $\mathrm{F}_{2}$ populations segregating for male sterility were selected for further genetic study.

SNP discovery by amplicon sequencing of $m s 5$ on A12 and ms6 on D12

Two recessive GMS gene, $m s 5$ and $m s 6$, were previously mapped to chromosomes 12 (A12) and 26 (D12), respectively, using SSR markers (Chen et al. 2009). The primer sequences of SSR markers linked to $m s 5$ and $m s 6$ were mapped to diploid $\mathrm{D}$ genome sequence of G. raimondii (JGI v2.0, annot v2.1, www. cottongen.org) by in silico comparative mapping approach. The diploid D genome sequence between $19,114,325$ bp and $21,205,044 \mathrm{bp}$ was used to discover genomic polymorphism linked to $m s 5$ and $m s 6$. PCR assays to produce sequencing templates were designed using Primer3 (http://frodo.wi.mit.edu/ primer3/) with default parameters. G. hirsutum lines including TM-1, DPGh98018 (male fertile) and DPGh04651 (GMS donor) and diploid A (G. arboreum, PI629477) and D (Gossypium raimondii, PI530898) as subgenomic references were used for amplicon PCR. To demonstrate homoeology between two loci on chromosome 12 and 26, respectively, chromosome nomenclature of A12 and D12 was used instead.

\section{PCR for sequencing and SNP genotyping}

PCR to amplify sequencing template was prepared using Platinum ${ }^{\circledR}$ Taq DNA Polymerase High Fidelity manufactured by Life Technologies (Grand Island, NY) following the manufacturer's manual. The PCR was conducted by initial denaturation at $95{ }^{\circ} \mathrm{C}$ for 5 min followed by 35 cycles of denaturation at $95^{\circ} \mathrm{C}$ for $1 \mathrm{~min}$, annealing at $55^{\circ} \mathrm{C}$ for $30 \mathrm{~s}$, and extension at $72{ }^{\circ} \mathrm{C}$ for $2 \mathrm{~min}$ and a final extension at $72{ }^{\circ} \mathrm{C}$ for 
5 min. Sequencing of PCR amplicons was performed using 3730XL DNA Analyzer (Life Technologies, Grand Island, NY), and sequence analysis and SNP discovery were conducted using CLC Bio Genomics Workbench software (Aarhus, Denmark). All genomic polymorphisms identified by amplicon sequence analysis were converted to TaqMan ${ }^{\circledR}$ assays for further SNP validation and SNP genotyping. DNA extraction was done by a protocol developed by Dellaporta et al. (1983).

PCR of $5 \mu \mathrm{l}$ total volume for SNP assay validation and SNP genotyping was conducted using GTXpress $^{\mathrm{TM}}$ Master Mix for SNP genotyping assay manufactured by Life Technologies following the manufacturer's manual (Life Technologies, Grand Island, NY). Each PCR included two PCR primers (final concentration of $900 \mathrm{nmol}$ for each primer), two TaqMan ${ }^{\circledR}$ MGB probes with NFQ labeled by FAM or VIC dye (final concentration of $250 \mathrm{nmol}$ for each probe) and $4 \mathrm{ng}$ genomic DNA. The PCR was conducted using GeneAmp ${ }^{\circledR}$ PCR System 9700 (Life Technologies, Grand Island, NY) by initial denaturation at $95{ }^{\circ} \mathrm{C}$ for $10 \mathrm{~min}$ followed by 40 cycles of denaturation at $95{ }^{\circ} \mathrm{C}$ for $15 \mathrm{~s}$, annealing and extension at $60{ }^{\circ} \mathrm{C}$ for $60 \mathrm{~s}$. SNP data collection and analysis were conducted by $\mathrm{ViiA}^{\mathrm{TM}} 7$ Software v1.2.1. (Life Technologies, Grand Island, NY).

\section{Genetic linkage of SNPs to $m s 5$ and $m s 6$}

$\mathrm{F}_{3}$ populations were generated by combining 14 different sibcross $F_{2}$ populations originated from a cross between DPGh98018 and DPGh04651. Genetic linkage between SNPs and chromosome assignment of linked markers to A12 or D12 were estimated by genetic distance calculated using R-Genetics (http:// cran.r-project.org/web/packages/genetics/genetics.pdf) among newly developed markers and also to the chromosome-specific markers assigned to A12 and D12 (Cho et al. 2014).

Fine mapping of both $m s 5$ and $m s 6$ was carried out by association analysis using a population of 1044 inbred lines representing 899 Monsanto germplasm lines of diverse genetic backgrounds consisting of 880 male fertile and 164 male sterile individuals. Diverse genetic materials were generated by unstructured random mating using male sterile progenies originated from DPGh04651 and diverse Monsanto germplasm lines. Male sterile phenotypes for each plant were binary coded as 1 for male fertile and 0 for male sterile and used in association analysis. Each plant was also genotyped by SNP markers putatively linked to $m s 5$ and $m s 6$, and two-step analysis was performed to identify haplotype marker set predicting male sterility. First, logistic regression was performed using binary sterility outcome with corresponding marker genotypes as predictors on the assumption of monogenic recessive model. As a second step, markers showing linkage to $m s 5$ or $m s 6$ loci were subjected to logistic regression analysis assessing relationships of alleles between two loci in association with the male sterility.

Haplotype SNPs associated with GMS were also tested on Lankart 57 (PI528822), Gregg (PI529094), Empire WR (PI529224) and G. tomentosum (PI 530723) which were reportedly served as donors of recessive $m s 5$ and $m s 6$ alleles (Weaver 1968), and the origins of recessive $m s 5$ and $m s 6$ alleles were estimated based on haplotype SNP patterns in each test material.

\section{Results}

Genetic characterization of $m s 5$ and $m s 6$ genes using mapping populations

Three independent sibcrossings between three different pairs of fertile and sterile $\mathrm{F}_{2}$ siblings produced sibcross $F_{1}$ progenies, and fertile-to-sterile phenotype ratios within these sibcross $F_{1}$ populations were close to 1:1 (Table 1). Based on 1:1 phenotype ratio within these

Table 1 Segregation of genetic male sterility in $F_{1}$ of $F_{3}$ sibling crosses and their $\mathrm{F}_{2}$ progenies

\begin{tabular}{lccll}
\hline Generation & $\begin{array}{l}\text { Fertile } \\
\text { frequency }\end{array}$ & $\begin{array}{l}\text { Sterile } \\
\text { frequency }\end{array}$ & $\begin{array}{l}\text { Expected } \\
\text { ratio (F:S) }\end{array}$ & $\begin{array}{l}\text { Chi-square } \\
\text { probability }\end{array}$ \\
\hline $\mathrm{F}_{1}^{\mathrm{a}}$ & 11 & 19 & $1: 1$ & 0.144 \\
$\mathrm{~F}_{1}^{\mathrm{a}}$ & 1 & 2 & $1: 1$ & 0.564 \\
$\mathrm{~F}_{1}^{\mathrm{a}}$ & 3 & 0 & $1: 1$ & 0.083 \\
$\mathrm{~F}_{2}^{\mathrm{b}}$ & 51 & 27 & $3: 1$ & 0.05 \\
$\mathrm{~F}_{2}^{\mathrm{b}}$ & 26 & 16 & $3: 1$ & 0.05 \\
$\mathrm{~F}_{2}^{\mathrm{b}}$ & 25 & 11 & $3: 1$ & 0.441 \\
$\mathrm{~F}_{2}^{\mathrm{b}}$ & 76 & 18 & $3: 1$ & 0.19 \\
$\mathrm{~F}_{2}^{\mathrm{b}}$ & 45 & 20 & $3: 1$ & 0.283 \\
$\mathrm{~F}_{2}^{\mathrm{b}}$ & 399 & 35 & $15: 1$ & 0.118 \\
\hline
\end{tabular}

${ }^{\text {a }}$ Sibcross $F_{1}$ progenies from a cross between fertile and sterile siblings selected from $\mathrm{F}_{3}$ segregating populations

${ }^{b} F_{2}$ progenies from $F_{1}$ by selfing 
small populations of sibling crosses, genotypes of fertile parent plants used in these crosses were speculated to be $M s 5 m s 5 m s 6 m s 6$ or ms $5 m s 5 M s 6 m s 6$, while sterile progenies were homozygous recessive for both loci. Fertile progenies from these sibcrosses were advanced to $F_{2}$ populations by selfing, and male sterility phenotypes were monitored. Segregation patterns of phenotypes in $\mathrm{F}_{2}$ populations were either 1:3 sterile to fertile indicating that a single gene was segregating or 1:15 sterile to fertile indicating that both genes were segregating in each $\mathrm{F}_{2}$ population (Table 1).

Sequencing to discover SNPs for $m s 5$ and $m s 6$

Whole-genome sequence of $G$. raimondii D genome (JGI v2.0, annot v2.1, www.cottongen.org) was used as a reference to nominate target regions for discovery of SNPs. Presumably about 0.5-Mbp-long sequences were subjected to amplicon sequencing. Sequence polymorphism was identified in forms of single-nucleotide polymorphism or single- or multiple-nucleotide deletion among the test materials which included male fertile and male sterile inbred lines on diploid A and D genome species. Among all genomic polymorphism identified within target regions covered by amplicon sequences, 23 polymorphic sequences qualified for PCR assay design were subjected to further analysis.

\section{Genetic linkage of SNPs to $m s 5$ and $m s 6$}

SNPs were tested for linkage to $m s 5$ on A12 and $m s 6$ on D12 using $F_{3}$ populations originated from 14 different sibcross $F_{2}$ populations segregating for male sterility. Polymorphisms of SNP markers corresponding to all 23 sequence polymorphisms identified by amplicon sequencing were tested in $\mathrm{F}_{3}$ populations, and genetic distances between SNPs and linkage to $m s 5$ and $m s 6$ were calculated based on genetic linkage to chromosome-specific SNPs. All 23 markers segregated in $\mathrm{F}_{3}$ sibcross populations, and all were assigned to $m s 5$ and $m s 6$ loci. Annotation of the putative genes carrying SNPs linked to $m s 5$ and $m s 6$ and their in silico comparative map positions on chromosome 08 on $G$. raimondii $\mathrm{D}$ genome (D5) are summarized in Table 2 and Fig. 1. Further analysis to associate each marker to the trait was conducted by the association mapping approach.
Association mapping to $m s 5$ and $m s 6$ loci

Monsanto germplasm lines of diverse genetic backgrounds consisted of 880 male fertile and 164 male sterile inbred lines were genotyped by all 23 SNPs assigned to $m s 5$ and $m s 6$. Male sterility phenotypes were collected from all association test materials and the phenotype data were categorized in binary format as either male sterile or male fertile. Using logistic regression model, all possible SNP combinations between two loci were tested to explain male sterility within diverse test materials. The recessive $m s 5$ allele was explained best by TT allele on MOGH583971 and TT allele on MOGH582973, and the recessive $m s 6$ allele was explained best by $\mathrm{CC}$ allele on MOGH211275 and GG allele on MOGH493571. When these two haplotypes for recessive alleles of $m s 5$ and $m s 6$ were combined, phenotype predictability in the diverse test panel was $99.6 \%$ (Table 3). By putative gene annotation, these markers are located on PHD-zinc-finger-like domain protein on A12 and AP2 gene on D12. Detailed SNP information for TaqMan genotyping is summarized in Table 4.

\section{Origin of $m s 5$ and $m s 6$}

Lankart 57 (PI528822), Gregg (PI529094), Empire WR (PI529224) and G. tomentosum were tested for the presence of $m s 5$ and $m s 6$ alleles using the haplotype markers described above. Based on haplotype patterns of the samples tested, G. tomentosum indicated the presence of $m s 5$ and $m s 6$ and Lankart 57 indicated the presence of $m s 6$. All other varieties tested showed no presence of the $m s 5$ and $m s 6$ haplotypes. From these results, it is probable that the $m s 5$ was transmitted from G. tomentosum. However, since none of the $\mathrm{F}_{2}$ populations occurring before the crossing with Lankart 57 showed male sterility (Weaver 1968), it is unlikely that the $m s 6$ allele from G. tomentosum was causative for male sterility. Therefore, the Lankart $57 \mathrm{~ms} 6$ gene is most likely responsible for male sterility in one of its $\mathrm{F}_{2}$ progenies in combination with $m s 5$ from $G$. tomentosum. It is also possible for the $m s 5$ allele of Lankart 57 to have differential phenotypic expression when compared to the $G$. tomentosum allele. 
Table 2 Summary of amplicon sequences used to identify genomic polymorphism at $m s 5$ on A12 and $m s 6$ on D12 and their in silico comparative map positions on D5 genome of Gossypium raimondii

\begin{tabular}{|c|c|c|c|}
\hline Chromosome & Sequence annotation & $\begin{array}{l}\text { Position on chromosome } 08 \\
\text { of D5 genome syntenic to } \\
\text { A12/D12 (base pairs) }\end{array}$ & Polymorphism \\
\hline $\mathrm{A} 12$ & Cytochrome p450 & $36601681-36601890$ & $\mathrm{C} / \mathrm{T}$ \\
\hline A12 & Cytochrome p450 & $36601598-36601781$ & $\mathrm{~A} / \mathrm{G}$ \\
\hline $\mathrm{A} 12$ & Cytochrome p450 & $36600316-36600453$ & $\mathrm{~A} / \mathrm{G}$ \\
\hline $\mathrm{A} 12$ & SINA protein family (zinc finger) & $36517055-36517264$ & $\mathrm{~A} / \mathrm{G}$ \\
\hline A12 & Ubiquitin family & $36513917-36513713$ & $\mathrm{~A} / \mathrm{G}$ \\
\hline A12 & Eukaryotic translation initiation factor 3 subunit & $36487438-36486837$ & $\mathrm{C} / \mathrm{T}$ \\
\hline A12 & PHD-zinc-finger-like domain & $36448804-36448648$ & $\mathrm{~A} / \mathrm{G}$ \\
\hline A12 & PHD-zinc-finger-like domain & $36448539-36448899$ & $\mathrm{C} / \mathrm{T}$ \\
\hline A 12 & PHD-zinc-finger-like domain & $36444422-36444671$ & $\mathrm{C} / \mathrm{T}$ \\
\hline A12 & Telomere stability and silencing & $36437252-36437058$ & $\mathrm{C} / \mathrm{T}$ \\
\hline A12 & Telomere stability and silencing & $36436860-36436639$ & $\mathrm{C} / \mathrm{T}$ \\
\hline A12 & Kinetochore centromere component & $36404821-36405030$ & $\mathrm{C} / \mathrm{G}$ \\
\hline A12 & Mitochondrial carrier-like protein & $36180644-36180843$ & $\mathrm{G} / \mathrm{T}$ \\
\hline D12 & Homeobox-associated leucine zipper & $36829091-36829251$ & $\mathrm{C} / \mathrm{T}$ \\
\hline D12 & ORDML family protein & $36823054-36823710$ & $\mathrm{C} / \mathrm{G}$ \\
\hline D12 & AP2(ERF domain) & $36670553-36670417$ & $\mathrm{C} / \mathrm{G}$ \\
\hline D12 & Cytochrome P450 & $36600741-36600580$ & $\mathrm{~A} / \mathrm{G}$ \\
\hline D12 & Cytochrome P450 & $36600741-36600580$ & $\mathrm{~A} / \mathrm{G}$ \\
\hline D12 & Aspartic proteinase & $36590566-36590776$ & $\mathrm{~A} / \mathrm{G}$ \\
\hline D12 & PHD-zinc-finger-like domain & $36443655-36443785$ & $\mathrm{G} / \mathrm{C}$ \\
\hline D12 & Cyclic nucleotide-gated ion channel & $36432550-36432377$ & $\mathrm{C} / \mathrm{T}$ \\
\hline D12 & Cyclic nucleotide-gated ion channel & $36432269-36432083$ & $\mathrm{C} / \mathrm{G}$ \\
\hline D12 & Cyclic nucleotide-gated ion channel & $36429252-36429459$ & $\mathrm{C} / \mathrm{G}$ \\
\hline
\end{tabular}

${ }^{a}$ Physical position of amplicon sequences on whole genome of Gossypium raimondii (JGI assembly v2.0, www.cottongen.org)

\section{Discussion}

Map positions of $m s 5$ and $m s 6$ were defined by Chen and his colleagues in 2009 using higher density SSR markers tested on backcrossing populations. By testing molecular marker patterns in Ms5ms5Ms6ms6 of male fertile and ms $5 m s 5 m s 6 m s 6$ of male sterile progenies, the authors were able to identify markers explaining dominant and recessive alleles of each gene. Markers found to be associated with the genes were further tested for genetic distance against other existing molecular markers. Using populations segregating for only one of the two genes with the second fixed to recessive homozygote is another effective approach beyond synthetic backcrossing population method used by Chen and his colleagues. However, the single gene segregating population approach will require tedious monitoring of progeny phenotypes in each family and can be very difficult to determine which gene is segregating in each population. In our study, we scanned entire genetic regions neighboring $m s 5$ and $m s 6$ and discovered markers polymorphic in diverse genetic backgrounds. All newly developed SNPs were quickly mapped to $m s 5$ and $m s 6$ by in silico comparative mapping using D diploid genome sequence and by linkage analysis using randomly segregating $\mathrm{F}_{3}$ populations. Based on known genetic locations and distances between markers, all possible pairwise combinations of markers between two different chromosomes were tested for association with male sterility using logistic regression modeling. As a result, two haplotypes consisting of 2 SNPs linked to 


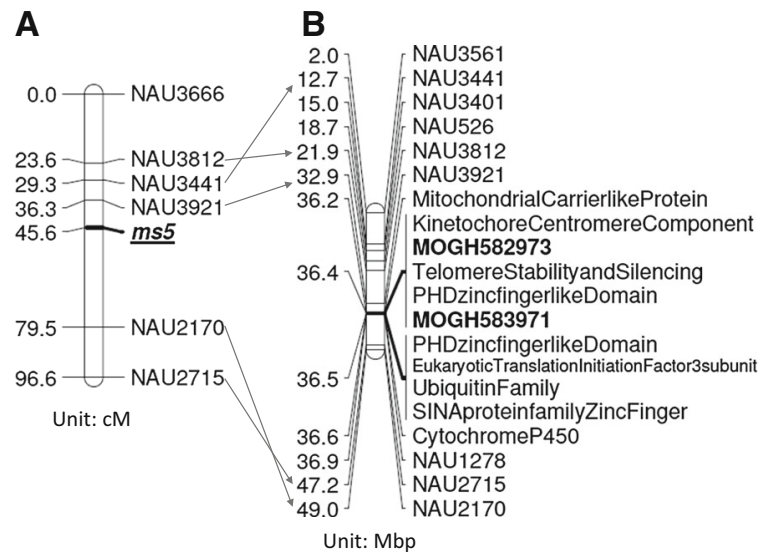

Fig. 1 In silico comparative mapping of molecular markers linked to $m s 5$ and $m s 6$ to chromosome 08 of G. raimondii D genome (D5). Maps a and $\mathbf{c}$ were recreated from the publication by Chen et al. (2009) describing map positions of $m s 5$ and $m s 6$ on chromosome 12 (A12) and 26 (D12), respectively. Maps $\mathbf{b}$ and $\mathbf{d}$ are summary of in silico comparative map positions of

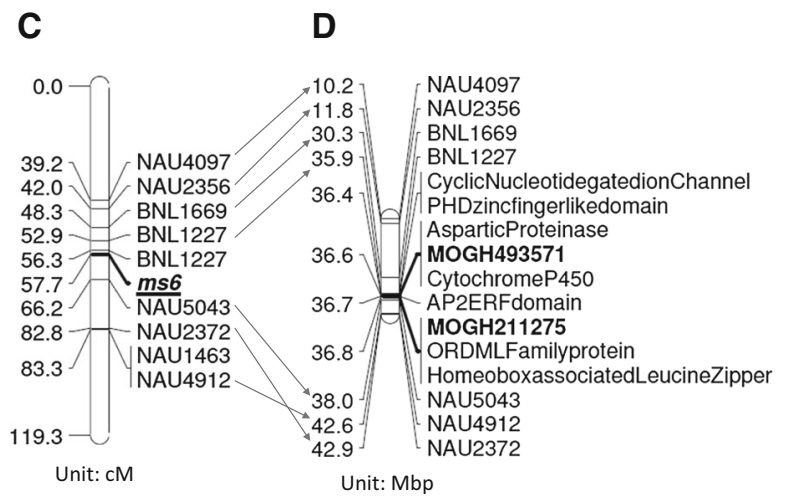

SSRs and newly developed SNPs linked to $m s 5$ and $m s 6$ on chromosome 08 of D5 genome. In silico map positions of SSRs on D5 genome are available in CottonGen (http://www. cottongen.org/gb/gbrowse/JGI_221_Dgenome/). Gene names on maps $\mathbf{b}$ and $\mathbf{d}$ represent annotation of the template sequences used to develop SNPs

Table 3 Probability of male sterility occurrence using genotype combinations of MONGH583971 (TT) and MONGH582973 (TT) associated with $m s 5 m s 5$ and MONGH211275 (CC) and MONGH493571 (GG) associated with ms6ms6

\begin{tabular}{lllll}
\hline Frequency (count) & $\begin{array}{l}\text { Individual with male } \\
\text { sterile haplotype on } \\
m s 5 \text { and } m s 6\end{array}$ & $\begin{array}{l}\text { Individual with male } \\
\text { fertile haplotype on } \\
m s 5 \text { and } m s 6\end{array}$ & $p$ value & Probability \\
\hline Male sterile individual & 109 & 17 & $2.00 \mathrm{E}-16$ & $99.60 \%$ \\
Male fertile individual & 1 & 769 & \\
\hline
\end{tabular}

$m s 5$ and 2 SNPs linked to $m s 6$ were identified that explained male fertility and sterility with $99 \%$ accuracy.

Two GMS genes, $m s 5$ and $m s 6$, were expected to be homoeologs and possibly duplicated through polyploidization. However, we found haplotype markers representing $m s 5$ and $m s 6$ located about $150 \mathrm{Kbp}$ apart from one another on the diploid D genome sequence. If genomic variation exist only on one of two homoeologs, then it is still possible to identify polymorphic markers unique to one homoeologous chromosome. Additional genomic polymorphisms at flanking regions from the other homoeologous chromosome can be used as flanking markers and allowing the two haplotypes high phenotypic prediction accuracy.

From the in silico comparative mapping of template sequences of the SNPs to diploid D genome, genomic regions targeted by male sterility haplotypes appeared to be very narrow and contained a limited number of genes. Based on putative gene annotation, PHD-zincfinger-like domain protein on $\mathrm{A} 12$ and AP2 (ERF domain) on D12 were contained between markers defining the haplotypes. Arabidopsis MALE STERILITY1 gene was found to be PHD-zinc-finger-like domain protein which plays a critical role in pollen development (Wilson et al. 2001; Ito et al. 2007). Recessive alleles created by EMS mutation conferred male sterility in Arabidopsis only when it is homozygous recessive. Pollens of $m s 1$ mutant with homozygous recessive alleles failed developing viable pollen (Wilson et al. 2001). The function of APETALA2 (AP2) as a negative regulator of AGAMOUS has been well established using Arabidopsis flower development mutants. Stamen, ovule and other floral organ development was significantly prohibited in the ap2 mutant (Jofuku et al. 1994). Further investigation is needed to prove functional association of any of these genes to male sterility in cotton. 
Table 4 Haplotype SNP set designed to predict genetic male sterility in Gossypium hirsutum conferred by paired recessive genes, $m s 5$ on $\mathrm{A} 12$ and $m s 6$ on D12

\begin{tabular}{|c|c|c|c|}
\hline Chromosome & SNP name & Polymorphism $^{\mathrm{a}}$ & $\begin{array}{l}\text { TaqMan SNP assay oligo sequence } \\
\left(5^{\prime} \rightarrow 3^{\prime}\right)\end{array}$ \\
\hline A12 & MOGH583971 & $\mathrm{C} / \mathrm{T}(m s 5)$ & $\begin{array}{l}\text { F: AGAACATTGATTATTACTGCCCCGAAT } \\
\text { R: GGCTCTCTTTTTGCTAAGCATGATT } \\
\text { P-FAM: CTTGGACTTCACTTTAC } \\
\text { P-VIC: CTTGGACTTCGCTTTAC }\end{array}$ \\
\hline A12 & MOGH582973 & $\mathrm{C} / \mathrm{T}(m s 5)$ & $\begin{array}{l}\text { F: CCGGTTCGGTTTATAAGAGAAGATCTG } \\
\text { R: AAATGTCTTTCTCTTCCTCGGAACC } \\
\text { P-FAM: CTCTCACTCAATACTC } \\
\text { P-VIC: ACTCTCACTCGATACTC }\end{array}$ \\
\hline D12 & MOGH211275 & $\mathrm{C}(m s \sigma) / \mathrm{G}$ & $\begin{array}{l}\text { F: AGCTAATGCTCTCTGACGGAACTA } \\
\text { R: ACAAAAGAGTAGATTTTAGTGTACCGTGTATTT } \\
\text { P-FAM: TGTGCGTAATTCATGATC } \\
\text { P-VIC: TTGTGCGTAATTGATGATC }\end{array}$ \\
\hline D12 & MOGH493571 & $\mathrm{A} / \mathrm{G}(m s \sigma)$ & $\begin{array}{l}\text { F: CTCTCGGGCCTGAACGA } \\
\text { R: GCTTTTACATCCCGGCTAAGACA } \\
\text { P-FAM: TGGGACGACGTTGAA } \\
\text { P-VIC: ATTATGGGACGATGTTGAA }\end{array}$ \\
\hline
\end{tabular}

" Single-nucleotide polymorphism linked to $m s 5$ and $m s 6$ was indicated by " $m s 5$ or $m s 6$ " designation

${ }^{\mathrm{b}}$ F, R, P-FAM and P-VIC represent forward primer, reverse primer and two probes labeled by FAM and VIC dyes, respectively. Oligo sequences for SNP markers and template sequences of $m s 5$ and $m s 6$ are available at CottonGen (http://www.cottongen.org/ search/markers)

The initial $m s 5$ and $m s 6$ study hypothesized the origin of the alleles from Gregg and Lankart 57. The reasoning is that following the identification of the first sterile individual, backcrossing was performed with Gregg and Lankart 57, each producing $\mathrm{F}_{2}$ segregating populations with 3:1 male-fertile-to-male-sterile ratio. Our study with haplotype markers tested on Lankart 57, Gregg, Empire WR and G. tomentosum showed that recessive $m s 5$ allele was present only in $G$. tomentosum and that recessive ms6 allele was present in G. tomentosum and Lankart 57. This indicates that $m s 5$ allele in male sterile $\mathrm{F}_{2}$ progeny was likely transmitted from G. tomentosum. However, due to absence of male sterile progenies in the $\mathrm{F}_{2}$ populations occurring before the crossing with Lankart 57 , it is likely that $m s 6$ allele causative for male sterility is found in Lankart 57 and not G. tomentosum. Although genotype test materials we used might not be identical to the ones used in the study by Weaver in 1968, the results from our study were able to explain potential sources of genetic male sterility in cotton.
In conclusion, a haplotype SNP marker set that consisted of four SNPs, two linked to $m s 5$ on A12 and two linked to ms6 on D12 in G. hirsutum was developed. This haplotype marker set was able to predict male sterile phenotype at the rate of $99 \%$ accuracy within our diverse germplasm test set. Each haplotype SNP marker can differentiate zygosity of $m s 5$ and $m s 6$ loci and can be used to predict GMS phenotype in G. hirsutum of diverse genetic background at any generations including inbred parents and progenies segregating randomly. With reliability as molecular markers and tight linkage to $m s 5$ and $m s 6$, our GMS haplotype SNP marker set can serve as a high-throughput molecular breeding tool to select GMS individuals to be used as hybrid parents and also to confirm purity of hybrids produced through larger scale commercial seed production. This new molecular breeding tool should be able to eliminate tedious hand emasculation and fertile plant removal after flower phenotyping in the field and improve genetic purity and quality of hybrid production. 
Conflict of interest The authors declare that they have no conflict of interest.

Open Access This article is distributed under the terms of the Creative Commons Attribution 4.0 International License (http:// creativecommons.org/licenses/by/4.0/), which permits unrestricted use, distribution, and reproduction in any medium, provided you give appropriate credit to the original author(s) and the source, provide a link to the Creative Commons license, and indicate if changes were made.

\section{References}

Aarts MG, Hodge R, Kalantidis K, Florack D, Wilson ZA, Mulligan BJ, Stiekema WJ, Scott R, Pereira A (1997) The Arabidopsis MALE STERILITY 2 protein shares similarity with reductases in elongation/condensation complexes. Plant J 12(3):615-623

Basu AK (1996) Current genetic research in cotton in India. Genetic 97:279-290

Bhale NL, Bhat MG (1990) Investigations on exploitation of heterosis in cotton (Gossypium hirsutum L.) using male sterility. Ind. J Genet 50(1):37-44

Chaudhury AM (1993) Nuclear genes controlling male fertility. Plant Cell 5(10):1277-1283

Chen D, Ding Y, Guo W, Zhang T (2009) Molecular mapping of genic male-sterile genes $m s 15, m s 5$ and $m s 6$ in tetraploid cotton. Plant Breed 128:193-198

Cho S, Yanai G, Schwarz J, Curtis A, Butruille M (2014) Genome-wide SNP marker panel applicable to cotton genetic diversity test. Proc Int Cotton Genome Initiat Conf 2(1):p11

Dellaporta S, Wood J, Hicks J (1983) A plant DNA mini preparation: version II. Plant Mol Biol Rep 1(4):19-21

Feng CD, Stewart JM, Zhang JF (2005) STS markers linked to the Rf1 fertility restorer gene of cotton. Theor Appl Genet 110(2):237-243

Gupta R, Ting JT, Sokolov LN, Johnson SA, Luan S (2002) A tumor suppressor homolog, AtPTEN1, is essential for pollen development in Arabidopsis. Plant Cell 14:2495-2507

Ito T, Nagata N, Yoshiba Y, Ohme-Takagi M, Ma H, Shinozaki K (2007) Arabidopsis MALE STERILITY1 encodes a PHDtype transcription factor and regulates pollen and tapetum development. Plant Cell 19(11):3549-3562

Jofuku KD, den Boer BG, Montagu MV, Okamuro JK (1994) Control of Arabidopsis flower and seed development by the homeotic gene APETALA2. Plant Cell 6(9):1211-1225

Justus NJ, Leinweber CL (1960) A heritable partial male-sterile character in cotton. J Hered 51(4):191-192

Justus NJ, Meyer JR, Roux JB (1963) A partially male-sterile character in upland cotton. Crop Sci 3(5):428-429

Liu L, Guo W, Zhu X, Zhang T (2003) Inheritance and fine mapping of fertility restoration for cytoplasmic male sterility in Gossypium hirsutum L. Theor Appl Genet 106(3):461-469

Meyer VG (1969) Some effects of genes, cytoplasm, and environment on male sterility of cotton (Gossypium). Crop Sci 9:237-242

Meyer VG (1973) Fertility restorer genes for cytoplasmic malesterility from Gossypium harknessii. In: Proc Beltwide Cotton Prod Res Conf, p 65

Meyer VG (1975) Male sterility from Gossypium harknessii. J Hered 66:23-27

Meyer VG, Meyer JR (1965) Cytoplasmically controlled male sterility in cotton. Crop Sci 5:444-448

Okada K, Shimura Y (1994) Genetic analyses of signaling in flower development using Arabidopsis. Plant Mol Biol 26(5):1357-1377

Preston J, Wheeler J, Heazlewood J, Li SF, Parish RW (2004) AtMYB32 is required for normal pollen development in Arabidopsis thaliana. Plant J 40(6):979-995

Rhyne CL (1991) Male-sterile ms5ms5ms6ms6 and ms8ms8ms9ms9. In: Proc Beltwide Cotton Prod Res Conf, pp 532-533

Richmond TR, Kohel RJ (1961) Analysis of a completely malesterile character in American upland cotton. Crop Sci 1(6):397-401

Schnable PS, Wise RP (1998) The molecular basis of cytoplasmic male sterility and fertility restoration. Trends Plant Sci 3(5):175-180

Steiner-Lange S, Unte US, Eckstein L, Yang C, Wilson ZA, Schmelzer E, Dekker K, Saedler H (2003) Disruption of Arabidopsis thaliana MYB26 results in male sterility due to non-dehiscent anthers. Plant J 34(4):519-528

Stewart JM (1992). A new cytoplasmic male sterile and restorer for cotton. In: Proc Beltwide Cotton Prod Res Conf, p 610

Wang F, Stewart JM, Zhang J (2007) Molecular markers linked to the $R f 2$ fertility restorer gene in cotton. Genome 50(9):818-824

Weaver JB (1968) Analysis of a genetic double recessive completely male-sterile cotton. Crop Sci 8(5):597-600

Wilson ZA, Zhang DB (2009) From Arabidopsis to rice: pathways in pollen development. J Exp Bot 60(5):1479-1492

Wilson ZA, Morroll SM, Dawson J, Swarup R, Tighe PJ (2001) The Arabidopsis MALE STERILITY1 (MS1) gene is a transcriptional regulator of male gametogenesis, with homology to the PHD-finger family of transcription factors. Plant J 28(1):27-39

Yin J, Guo W, Yang L, Liu L, Zhang T (2006) Physical mapping of the $R f 1$ fertility-restoring gene to a $100 \mathrm{~kb}$ region in cotton. Theor Appl Genet 112(7):1318-1325

Zhang JF, Stewart JM (2001) Inheritance and genetic relationship of the D8 and D2-2 restorer genes for cotton cytoplasmic male sterility. Crop Sci 41(2):289-294

Zhang JF, Stewart JM (2004) Identification of molecular markers linked to the fertility restorer genes for CMS-D8 in cotton (Gossypium hirsutum L.). Crop Sci 44(4):1209-1217 